\title{
Multiple Electrophoretic Forms of Transaldolase and 6- Phosphogluconic Dehydrogenase and Their Relationships to the Taxonomy and Ecology of the Bifidobacteria
}

\author{
V. SCARDOVI, F, CASALICCHIO, AND N. VINCENZI \\ Istituto di Microbiologia Agraria, Università di Bologna, Bologna, Italy
}

\begin{abstract}
The electrophoretic patterns of the transaldolases and 6-phosphogluconate dehydrogenases (6PGD) of 1,206 strains, each belonging to one of 24 named species of the genus Bifidobacterium, were determined by means of starch-gel electrophoresis. All of these strains were previously assigned to species on the basis of their deoxyribonucleic acid homology relationships and were selected so as to include all known phenotypes and habitats. Fourteen electrophoretic forms of transaldolase and 19 of nicotinamide adenine dinucleotide phosphate-dependent 6PGD were identified and numbered. Each strain displayed one band for each enzyme. Glucose-grown cells of the B. pullorum strains and of most of the $B$. dentium strains were devoid of detectable levels of 6 PGD. The zymograms of more than $60 \%$ of the strains studied were species specific. Nearly half of the other strains had significant overlapping of transaldolase and 6PGD patterns, and they were assigned therefore to species on the basis of an additional marker, 3phosphoglyceraldehyde dehydrogenase production, the results of which are not reported here in detail. Of the species studied, B. asteroides exhibited the greatest variability in electrophoretic types of both transaldolase and 6PGD. Correlations between electrophoretic data, deoxyribonucleic acid homology relationships between the species, and ecology are discussed.
\end{abstract}

Fructose 6-phosphate phosphoketolase (F6PPK) is the key enzyme of carbohydrate dissimilation in the genus Bifidobacterium. Although its relative electrophoretic mobility is generally not a useful character for species differentiation, there are some differences in the properties of F6PPK in some Bifodobacterium species generally confined to specific habitats. The most anodic form of F6PPK was from $B$. asteroides (from the honey bee intestine); a second, less mobile F6PPK was from $B$. globosum, B. thermophilum, and B. suis (commonly isolated from the feces of various animals); a third F6PPK isozyme of intermediate electrophoretic mobility was present in $B$. breve, $B$. longum, and $B$. adolescentis (human strains from intestine, dental caries, and vagina) (24). A comparative study of the F6PPKs from the type strains of $B$. globosum and B. dentium disclosed that the enzyme from $B$. globosum was more heat stable than that from $B$. dentium, that the optimum activity was at $\mathrm{pH} 5$ to 6 for $B$. globosum and at $\mathrm{pH} 7$ for $B$. dentium, and that the "animal" type of F6PPK (from B. globosum) was activated by $\mathrm{Mg}^{2+}$ and was less active in the presence of $\mathrm{Mn}^{2+}$, whereas the reverse was true with the "human" type of F6PPK (from $B$. dentium), where only $\mathrm{Mn}^{2+}$ fully activated the enzyme. The molecular weights were different $\left(1.6 \times 10^{5}\right.$ and $2.9 \times 10^{5}$, respectively), and the enzyme from $B$. globosum also cleaved xylulose 5-phosphate, whereas the $B$. dentium F6PPK was specific for fructose 6-phosphate (33).

A preliminary study on the electrophoretic forms of other enzymes, such as transaldolase, transketolase, 6-phosphogluconate dehydrogenase (6PGD), and aldolase, was made with 49 representative strains of Bifidobacterium species; several isozymes were detected, but no clear correlation with the habitats was found (23). In the meanwhile, a vast number of bifidobacteria from diverse habitats (feces of animals under different diets, breast- or bottle-fed infants, sewage, etc.) were studied in our laboratory and were identified mainly by means of deoxyribonucleic acid (DNA)-DNA hybridization. Four additional "homology groups" were recognized and proposed as new species (28). Because of the disposal of a large number of strains of all currently known Bifidobacterium species, we thought it advisable to enlarge our electrophoretic studies to cover the largest possible number of Bifidobacterium strains as a start in exploring the internal structure of the genus.

The two enzymes dealt with in this study, transaldolase and 6PGD, are undoubtedly of different importance in the carbohydrate metabolism of bifidobacteria. Transaldolase, an essen- 
tial enzyme in the "fructose 6-phosphate shunt," is characteristic of bifidobacteria because it acts upon fructose 6-phosphate and erythrose 4phosphate after the initial formation of the latter from the former by a phosphoketolase; 6PGD is apparently a functionless enzyme, at least in glucose-grown cells, which, as a rule, lack detectable glucose 6-phosphate dehydrogenase (4, 25 ). Different extents of variability in the two enzymes can reasonably be expected during the course of bifidobacterial evolution (e.g., see Gillespie and Kojima [6]). A preliminary immunological study on the structural relatedness of bifidobacterial transaldolases has recently been performed with the ultimate goal of elucidating the evolutionary relationships of these organisms (34). The work described here is an extension of this study.

\section{MATERIALS AND METHODS}

Bacterial strains. The Bifidobacterium strains used in this study are listed in Table 1 . The two DNA homology groups described by Scardovi and Trovatelli (27) are referred to as "minimum" and "subtile" pending their formal proposal as new species.

Cell-free extracts. Cells of most of the strains were grown on Trypticase-phytone-yeast extract medium (TPY), of which the composition is reported elsewhere (22). The substrate $(900 \mathrm{ml}$ in 1-liter conical flasks) was sterilized at $110^{\circ} \mathrm{C}$ for 30 min, gassed with carbon dioxide passed through a porous glass disk for $1 \mathrm{~min}$ (final $\mathrm{pH}, 6.2$ to 6.4), and inoculated with $100 \mathrm{ml}$ of culture. B. magnum strains were grown in TPY medium enriched with Tween 80 (1 ml/liter). B. pullorum strains were grown on MRS medium (3). $B$. asteroides strains were grown on TPY medium $(\mathrm{pH}$ 7.0) which had been gassed with $\mathrm{CO}_{2}$ and which was contained in completely filled cylindrical 1-liter vessels. After 18 to $20 \mathrm{~h}$ of incubation at $39^{\circ} \mathrm{C}$, the cells were harvested, washed with phosphate buffer $(0.05$ $\mathrm{M}, \mathrm{pH} 6.5$ ), and disrupted by sonication. The extracts, clarified by centrifugation at $20,000 \mathrm{rpm}$ for $30 \mathrm{~min}$ in the cold, had a protein content varying from 5 to 25 $\mathrm{mg} / \mathrm{ml}(12)$.

Starch-gel electrophoresis. The starch-gel horizontal electrophoretic system of Smithies was used (36). Hydrolyzed starch (Connaught Medical Research Labs., Toronto, Canada) was used at a concentration of $90 \mathrm{~g}$ /liter of the appropriate buffer (see below). Plastic frames $(12.0$ by 37.0 by $0.9 \mathrm{~cm}$ or 6.0 by 37.0 by $0.9 \mathrm{~cm}$ ) were used to hold the slabs. Generally, 5 to 10 $\mu \mathrm{l}$ of extract (or dilution thereof) on Whatman 3MM paper strips $(0.5$ by $0.5 \mathrm{~cm})$ was run in the cold for 15 to $20 \mathrm{~h}$ with a current of 15 to $20 \mathrm{~mA}$ for transaldolase and 40 to $50 \mathrm{~mA}$ for 6PGD. Particular sets of extracts were used as references throughout this study (see below). Results were recorded photographically.

The following buffers were used. Transaldolasebridge buffer (per liter): tris(hydroxymethyl)-amino methane, $16.3 \mathrm{~g}$; citric acid monohydrate, $9.0 \mathrm{~g}(\mathrm{pH}$ 7.0); gel buffer: bridge buffer diluted 1:15. 6PGDbridge buffer: $0.4 \mathrm{M}$ monosodium citrate ( $\mathrm{pH} 7.0$ with citric acid) (this solution was renewed every two runs); gel buffer: $0.005 \mathrm{M}$ histidine plus $1.5 \mathrm{~g}$ of sodium chloride per liter (pH 7.0) (see ref. 1).

Staining procedures. (i) Transaldolase. The developing solution contained (per $100 \mathrm{ml}$ ): fructose 6phosphate (Na salt, $98 \%$ purity, Sigma), $400 \mathrm{mg}$; sodium arsenate, $370 \mathrm{mg}$; glycine, $240 \mathrm{mg}$; nicotinamide adenine dinucleotide, $13 \mathrm{mg}$; D-erythrose 4-phosphate (60 to $75 \%$ purity, Sigma), $16 \mathrm{mg}$; phenazine methosulfate, $2 \mathrm{mg}$; Nitro Blue Tetrazolium (Sigma), $20 \mathrm{mg}$; and about $130 \mathrm{U}$ of glyceraldehyde 3-phosphate dehydrogenase; the solution was adjusted to $\mathrm{pH} 7.0$.

(ii) 6PGD. Tris(hydroxymethyl) aminomethane-hydrochloride buffer $(0.5 \mathrm{M})$ ( $\mathrm{pH} 7.0), 10 \mathrm{ml}$; 6-phosphogluconic acid (trisodium salt, Sigma), $250 \mathrm{mg}$; nicotinamide adenine dinucleotide phosphate, $20 \mathrm{mg}$; $\mathrm{Ni}$ tro Blue Tetrazolium, $20 \mathrm{mg}$; phenazine methosulfate, $2 \mathrm{mg}$; and water, $90 \mathrm{ml}$.

(iii) 3-Phosphoglyceraldehyde dehydrogenase. The staining solution contained (per $100 \mathrm{ml}$ ): DL-glyceraldehyde-3-phosphate (prepared from diethylacetal, barium salt; Sigma), $200 \mathrm{mg}$ (neutralized solution from Dowex 50); $0.5 \mathrm{M}$ tris(hydroxymethyl)aminomethanehydrochloride buffer, (pH 8.5), $20 \mathrm{ml}$; sodium arsenate, $370 \mathrm{mg}$; phenazine methosulfate, $2 \mathrm{mg}$; Nitro Blue Tetrazolium, $20 \mathrm{mg}$; and nicotinamide adenine dinucleotide, $13 \mathrm{mg}$.

For staining, the starch slabs were flooded with 25 $\mathrm{ml}$ of the appropriate staining solution and kept at $37^{\circ} \mathrm{C}$ until discernible color developed (from $15 \mathrm{~min}$ to 1 to $2 \mathrm{~h}$ ). The slabs were washed, covered with a plastic sheet, and kept at 4 to $5^{\circ} \mathrm{C}$. During this storage, the zymograms gained in color and sharpness.

\section{RESULTS}

General characters of the zymograms and numbering of the isozymes. The ranges of the electrophoretic mobilities of the enzymes in the species examined are shown in Fig. 1 and 2 . The distances travelled by the fastest and by the slowest moving bands were sufficiently different to permit a clear-cut recognition of all the isozymes detected in this study, namely, 14 of transaldolase and 19 of 6PGD (see Fig. 3). The fastest moving (i.e., most anodic) band was designated by the number 1 , and the slowest (i.e., least anodic) was designated by the number 10 for transaldolase and 13 for 6PGD. The zymograms presented as Fig. 1 and 2 summarize an advanced, but not the final, stage of the present study; additional isozymes were found in the species more extensively investigated later: $B$. asteroides, B. indicum, B. boum, and B. thermophilum. It was thought desirable to refer to these additional isozymes by lower-case letters suffixed to the numbers already used.

Each strain exhibited only one band for either enzyme; in the few cases where two distinct bands were observed, mixed cultures, ascertained by subcloning and successive electrophoresis, were involved. Some species, for example $B$. asteroides, showed a tendency to give trailed 
TABLE 1. Source and electrophoretic forms of the transaldolase and 6PGD isozymes of the strains studied

$\begin{array}{lll}\text { Strains } & \text { Electrophoretic form of } \\ & \text { Source } & \text { Transaldolase } 6 \text { PGD }\end{array}$

Bifidobacterium cuniculi Scardovi et al.

Transaldolase $\quad$ 6PGD

RA93 (= ATCC 27916), Type $\operatorname{strain}(28) \ldots \ldots \ldots \ldots \ldots \ldots \ldots \ldots \ldots$ Feces of rabbit

RA94:RA95,RA98:RA99;RA104;RA105 ............. Feces of rabbit

Bifidobacterium pullorum Trovatelli et al.

P145 (= ATCC 27685), Type strain (37);P182;P183 .......... Feces of chicken

4

Rumen of cattle

Rumen of cattle

Rumen of cattle

Rumen of cattle

Feces of piglet

Feces of rat

Feces of rat

Feces of rat

Feces of rat

Feces of rat

Feces of lamb

Feces of lamb

Feces of chicken

Feces of chicken

Feces of chicken

Feces of rabbit

Feces of rabbit

Feces of rabbit

Feces of rabbit

Feces of calf

Feces of calf

Sewage

Feces of infant

absent

6

6

6

5

$3 \mathrm{a}$

6

5

6

F229;F387;F461;F468 ${ }^{2}$;F512;F544 ${ }^{d} ; \mathrm{F} 574 ; \mathrm{F} 591 ; \mathrm{F} 593 ; \mathrm{F} 607 \ldots \ldots \ldots$

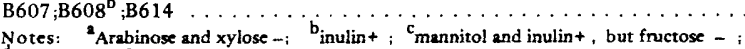

Notes:
$\mathrm{d}_{\text {fructose }}-; \quad$ e reported as B. pseudolongum (39)

Bifidobacterium pseudolongum Mitsuoka

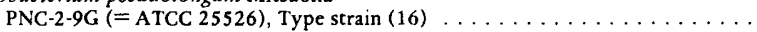

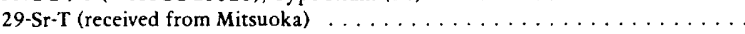

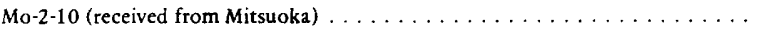

"Subtile" DNA homology group (27)

F395 (= ATCC 27537), Reference strain; F340;F394;F396;F398 . . . . . Sewage

Bifidobacterium choerinum Scardovi et al.

SU806 (=ATCC 27686), Type strain (28);SU827;SU836;SU837;SU838 . . .

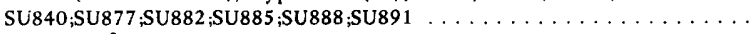

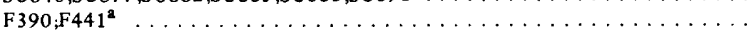

Note: ${ }^{2}$ Xylose not fermented.

Bifidobacterium pseudocatenulatum Scardovi et al.

B 1279 (= ATCC 27917), Type strain (28); B1280;B1286;B1330;B1336;B1342

$\mathrm{B} 1457 ; \mathrm{B} 1460 ; \mathrm{B} 1555 ; \mathrm{B} 1663 ; \mathrm{B} 1666 ; \mathrm{B} 1669 ; \mathrm{B} 1670 ; \mathrm{B} 1735$;B1889;B1957;B1960 $\mathrm{B} 1961 ; \mathrm{B} 1965 ; \mathrm{B} 1969 ; \mathrm{B} 1970 ; \mathrm{B} 1977 ; \mathrm{B} 2072 ; \mathrm{B} 2073 ; \mathrm{B} 2077 ; \mathrm{B} 2078 ; \mathrm{B} 2282 ; \mathrm{B} 2284$ $\mathrm{B} 2286 ; \mathrm{B} 2351 ; \mathrm{B} 2359 ; \mathrm{B} 2361 ; \mathrm{B} 2445 ; \mathrm{B} 2446 ; \mathrm{B} 2540 ; \mathrm{B} 2541 ; \mathrm{B} 2544 ; \mathrm{B} 2545$ $\mathrm{B} 1830^{\mathrm{a}} ; \mathrm{B} 1832^{\mathrm{a}} ; \mathrm{B} 1834^{\mathrm{a}} ; \mathrm{B} 1874 ; \mathrm{B} 1875 ; \mathrm{B} 1879 ; \mathrm{B} 1880 ; \mathrm{B} 1886 ; \mathrm{B} 1888 ; \mathrm{B} 2067 \ldots$

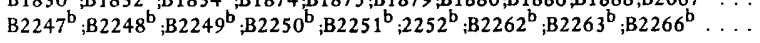
$\mathrm{B} 2339 ; \mathrm{B} 2340 ; \mathrm{B} 2650 ; \mathrm{B} 2652 ; \mathrm{B} 2654 ; \mathrm{B} 2656$

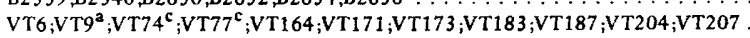
VT254;VT265;VT270;VT278;VT279;VT287;VT337;VT 339;VT341;VT348 . . VT351;VT 353;VT354;VT360;VT368;VT370;VT373

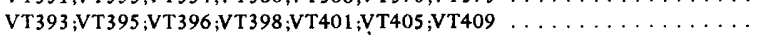
F54;F72;F262;F331;F356;F360;F469 d

Additional strains $^{e}$ : $\mathrm{F} 18 ; \mathrm{F} 19 \mathrm{F71} ; \mathrm{F} 101 ; \mathrm{F} 165 ; \mathrm{F} 166 ; \mathrm{F} 171 ; \mathrm{F} 177 ; \mathrm{F} 209 \ldots \ldots$

$\mathrm{F} 240 ; \mathrm{F} 335 ; \mathrm{F} 344 ; \mathrm{F} 400 ; \mathrm{F} 469$

Notes: ${ }^{2}$ Mannitol +; bonly $60 \%$ related to B.pseudocatenulatum and B. catenulatum reference strains (Table 2, ref. 28); strains B2262 and B2266 were arabinose - ; while B2262 and B2263 and B2266 were xylose -; ' see Table 3, ref. 28, for their homology data; formerly regarded as a member of $B$. catenulatum (Table 1, ref.22) ${ }^{e}$ diagnosis based on sugar fermentation (starch+ ; see footnote to Table 6, ref. 28); other differences in sugar fermented reported eisewhere (28).

Bifidobacteriunt de'utium Scardovi and Crociani B764 (= ATCC 27534), Type strain (22)

Nelezitose positive ${ }^{\mathrm{a}}: \mathrm{B} 698 ; \mathrm{B} 709 ; \mathrm{B} 767^{\mathrm{C}} ; \mathrm{B} 774 ; \mathrm{B} 777 ; \mathrm{B} 968 ; \mathrm{B} 971 ; \mathrm{B} 973$ $\mathrm{B} 974 ; \mathrm{B} 977$ :B980;B982;B984;B986;B987;B992;B994;B1004;B1006;B1007 $\mathrm{B} 1012 ; \mathrm{B} 1013 ; \mathrm{B} 1014 ; \mathrm{B} 1015 ; \mathrm{B} 1016 ; \mathrm{B} 1017 ; \mathrm{B} 1019 ; \mathrm{B} 1023 ; \mathrm{B} 1025 ; \mathrm{B} 1027 ; \mathrm{B} 103]$ $\mathrm{B} 1032 ; \mathrm{B} 1033 ; \mathrm{B} 1035 ; \mathrm{B} 1036 ; \mathrm{B} 1038 ; \mathrm{B} 1043 ; \mathrm{B} 1046 ; \mathrm{B} 1049 ; \mathrm{B} 1051 ; \mathrm{B} 1053$;B1054 $\mathrm{B} 1056 ; \mathrm{B} 1057 ; \mathrm{B} 1058 ; \mathrm{B} 1061 ; \mathrm{B} 1064 ; \mathrm{B} 1067 ; \mathrm{B} 1069 ; \mathrm{B} 1080 ; \mathrm{B} 1081 ; \mathrm{B} 1086 ; \mathrm{B} 1090^{\mathrm{d}}$ $\mathrm{B} 1091 ; \mathrm{B} 1093^{\mathrm{d}} ; \mathrm{B} 1096^{\mathrm{d}} ; \mathrm{B} 1105^{\mathrm{d}} ; \mathrm{B} 1106^{\mathrm{d}} ; \mathrm{B} 1107 ; \mathrm{B} 1109^{\mathrm{d}} ; \mathrm{B} 1117 ; \mathrm{B} 1119 ; \mathrm{B} 1121$
Feces of pig

Feces of chicken

Feces of mouse

Feces of piglet Feces of piglet Sewage

Feces of infant Feces of infant Feces of infant Feces of infant Feces of infant Feces of infant Feces of infant Feces of calf Feces of calf Feces of calf Feces of calf Sewage Sewage Sewage 
TABLE 1-continued

\begin{tabular}{|c|}
\hline Strains \\
\hline $\begin{array}{l}\mathrm{B} 1123, \mathrm{~B} 1127 ; \mathrm{B} 1128 ; \mathrm{B} 1129 ; \mathrm{B} 1130 ; \mathrm{B} 1131 ; \mathrm{B} 1137 ; \mathrm{B} 1145 ; \mathrm{B} 1151 ; \mathrm{B} 1154 ; \mathrm{B} 1156 . \\
\mathrm{B} 1022 ; \mathrm{B} 1040 ; \mathrm{B} 1065 ; \mathrm{B} 1071, \mathrm{~B} 1074 ; \mathrm{B} 1142 ; \mathrm{B} 1148 \ldots \ldots \ldots \ldots \ldots\end{array}$ \\
\hline 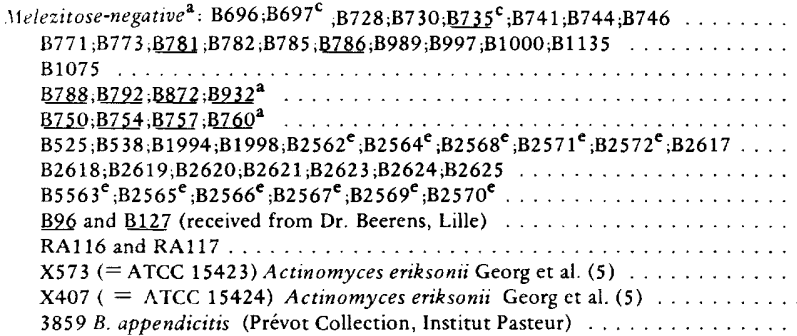 \\
\hline $\begin{array}{l}\text { Notes: }{ }^{a} \text { Only the underlined strains were used for DNA-DNA hybridization (22); } \\
\mathrm{b}_{\text {all of the strains listed were used for DNA-DNA hybridization (unpublished); }} \\
c_{\text {sorbitol }+;} d_{\text {inulin }+;} \text { eisolated from the same fecal specimen; indeterminable with spot } \\
\text { staining. }\end{array}$ \\
\hline
\end{tabular}

Source

Electrophoretic form of

Transaldolase

6PGD

Bifidobacterium magnum Scardovi and Zani

RA3 (= ATCC 27540), Type strain (31)

RA1;RA9:RA10;RA77;RA78;RA89;RA184;RA185:RA189;RA190.... Feces of rabbit

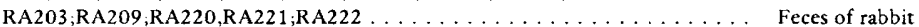

Dental caries

Dental caries

Dental caries

Dental caries

Dental caries

Human vagina

Feces of adult

Feces of infant ${ }^{b}$

Feces of infant

Feces of infant ${ }^{b}$

Oral cavity

Feces of rabbit

Man lung abscess

Man pleural fluid

Human appendix

Bifidobacterium catenulatum Scardovi and Crociani

B669 (= ATCC 27539), Type strain (22).

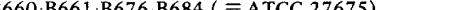

........ Feces of adult

$B 1331^{a} ; B 1333^{a} ; B 1334^{a} ; B 1338^{a} \cdot B 1341^{a} B 1738 ; B 1748^{2} ; B 1955 ; B 2218 \ldots \ldots$ Feces of infant

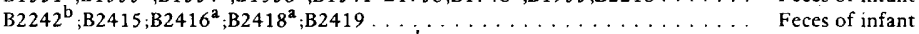

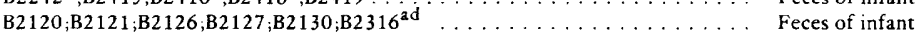

$\mathrm{F} 64 ; \mathrm{F} 94^{\mathrm{c}} ; \mathrm{F} 134 ; \mathrm{F} 141 ; \mathrm{F} 189^{\mathrm{b}} ; \mathrm{F} 217 ; \mathrm{F} 258 ; \mathrm{F} 276 ; \mathrm{F} 290 ; \mathrm{F} 291 ; \mathrm{F} 294 \ldots \ldots \ldots$ Sewage

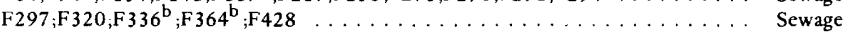

Notes: ${ }^{a}$ Fructose - ; ${ }^{b}$ mannitol + (F336 also sorbitol - ); ${ }^{c}$ xylose $-;{ }^{d}$ strains B2120 to B2130 from a single specimen, strain B2316 was isolated from the same individual 30 days later.

Bifidobacterium animalis (Mitsuoka) Scardovi and Trovatelli

R101-8 (= ATCC 25527), Type strain (27)

$\mathrm{T}^{\mathrm{ac}} ; \mathrm{T} 27^{\mathrm{ac}}, \mathrm{T} 98^{\mathrm{b}} ; \mathrm{T} 160^{\mathrm{ac}} ; \mathrm{T} 169$

$\mathrm{P} 16 ; \mathrm{P} 17 ; \mathrm{P} 23^{\mathrm{a}}(=\mathrm{ATCC} 27536) ; \mathrm{P} 32^{\mathrm{c}} ; \mathrm{P} 39^{\mathrm{c}} ; \mathrm{P} 45^{\mathrm{b}}$

RA12;RA13;RA14 ${ }^{\mathrm{C}}$ :RA15;RA16 ${ }^{\mathrm{c}}$;RA18;RA20;RA23 ${ }^{\mathrm{bc}}$

F337;F349;F437;F439

Notes: ${ }^{a}$ Mannose +; ${ }^{b}$ cellobiose $-;{ }^{c}$ melezitose +

Bifidobacterium anyulatum Scardovi and Crociani

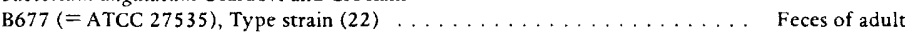

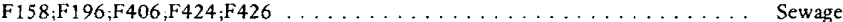

Feces of rat

Feces of rat

Feces of chicken

Feces of rabbit

Sewage

$\begin{array}{lc}4 & \text { indet. } \\ 5 & \text { indet. } \\ 4 & \text { indet. } \\ 4 & \text { indet. } \\ 5 & \text { indet. } \\ 4 & \text { indet. } \\ 4 & \text { indet. } \\ 4 & 2 \\ 4 & 2 \\ 5 & 2 \\ 4 & 2 \\ 4 & \text { indet. } \\ 4 & 2 \\ 4 & 2 \\ 4 & \text { indet. }\end{array}$

7

6

6

8

6

ATC 27920), Reference for DNA homology grouping ... Feces of infant

S76e (= ATCC 15702), Type strain B. liberorum Reuter $(20) \ldots \ldots \ldots \ldots$ Feces of infant

$659(=$ ATCC 25962$)$, Type strain B. lactentis Reuter $(20) \ldots \ldots \ldots \ldots$ Feces of infant

Feces of infant

Pentoses-negative strains (like strain $\mathrm{S} 12$ )

$\mathrm{B} 625^{\mathrm{b}} ; \mathrm{B} 642^{\mathrm{b}} ; \mathrm{B} 649^{\mathrm{b}} ; \mathrm{B} 651$

Feces of infant

9

9
8

8

8

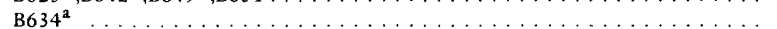

Pentoses positive strains

B135i;B1 378;B1412;B1436 ${ }^{\mathrm{d}} ; \mathrm{B} 1490 ; \mathrm{B} 1509 ; \mathrm{B} 1528 ; \mathrm{B} 1599 ; \mathrm{B} 1602 ; \mathrm{B} 1635^{\mathrm{e}}$

Feces of infant $\mathrm{B} 1638 ; \mathrm{B} 1648 ; \mathrm{B} 1652 ; \mathrm{B} 1654 ; \mathrm{B} 1658 ; \mathrm{B} 1673^{\mathrm{f}} ; \mathrm{B} 1707 ; \mathrm{B} 1709 ; \mathrm{B} 1717 ; \mathrm{B} 1719 \ldots .$. Feces of infant $\mathrm{B} 1721 ; \mathrm{B} 1730 ; \mathrm{B} 1731 ; \mathrm{B} 1732 ; \mathrm{B} 1799 ; \mathrm{B} 1805 ; \mathrm{B} 1822 ; \mathrm{B} 1824 ; \mathrm{B} 1851 ; \mathrm{B} 1853 \ldots \ldots$ Feces of infan $\mathrm{B} 1854 ; \mathrm{B} 1858 ; \mathrm{B} 1860 ; \mathrm{B} 1866^{\mathrm{h}} ; \mathrm{B} 1882 ; \mathrm{B} 1883 ; \mathrm{B} 1915 ; \mathrm{B} 1918 ; \mathrm{B} 1922 ; \mathrm{B} 1934 \ldots \ldots$ Feces of infant B1939;B1948;B1951;B1954;B1956;B2016;B2026;B2032;B2037;B2042 . . . . Feces of infant

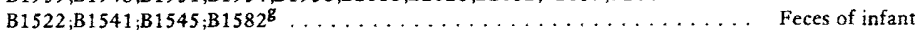

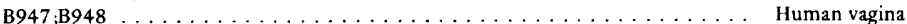
Notes: ${ }^{a}$ Unique strain melezitose and gluconate + ; $b$ mannitol+ (like strain 659), B649 is also sorbitol $t_{;}{ }^{c}$ melezitose, mannitol, sorbitol and starch -; ${ }_{\text {same specimen }}$ as B1427, B1429 and 1434 (see under $B$. longum), melezitose $+;{ }^{C}$ same specimen

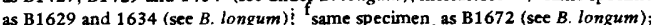

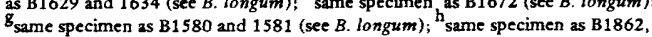
$B 1864$ and $B 1872$ (see B. longum). 
TABLE 1-continued

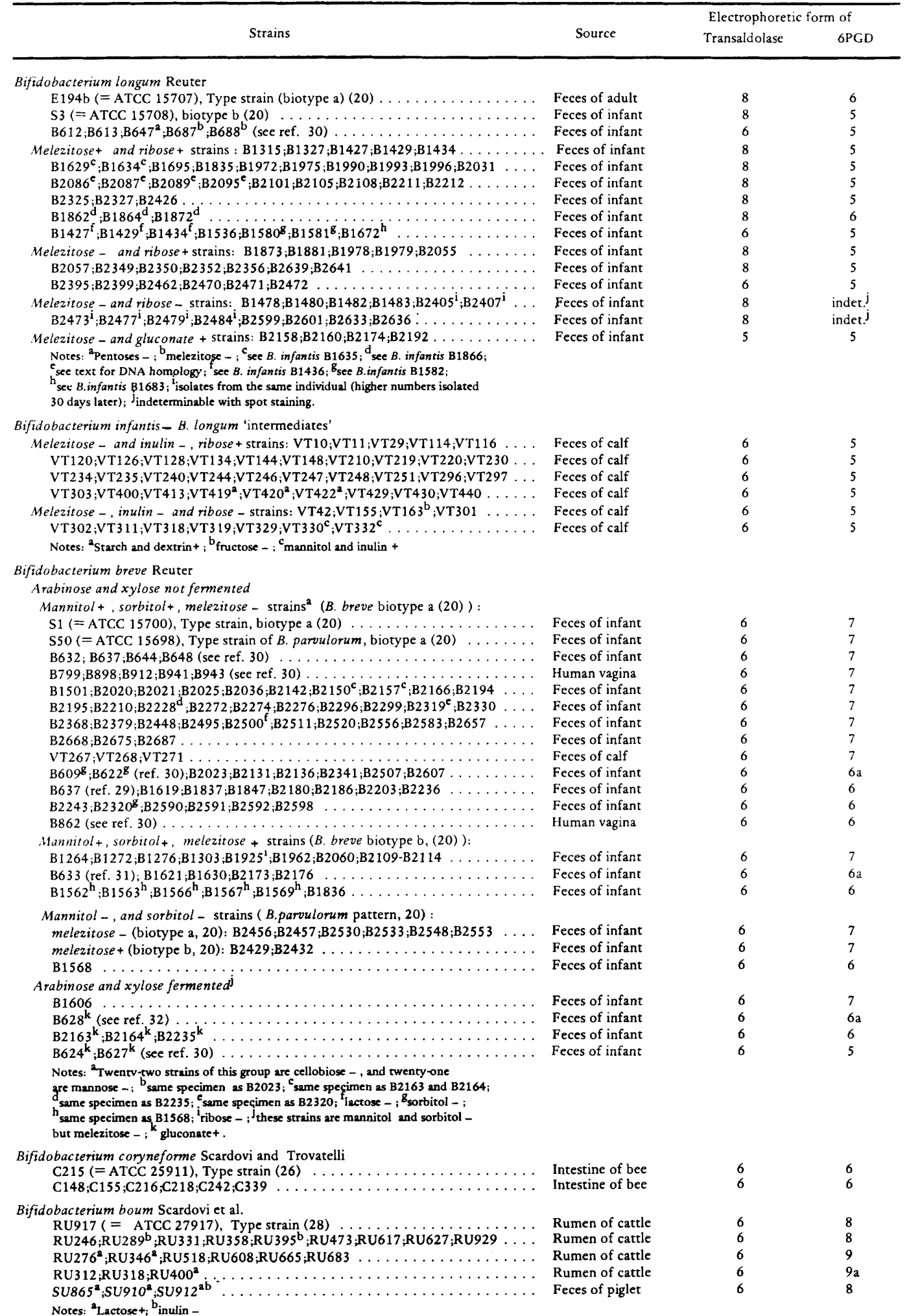


TABLE $1-$ continued

\begin{tabular}{|c|c|c|c|}
\hline \multirow{2}{*}{ Strains } & \multirow[b]{2}{*}{ Source } & \multicolumn{2}{|c|}{ Electrophoretic form of } \\
\hline & & Transaldolase & $6 \mathrm{PGD}$ \\
\hline
\end{tabular}

Bifidobacterium suis Matteuzzi et al.

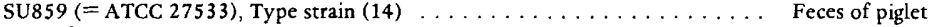

SU864 ${ }^{\text {; }}$ SU868 (= ATCC 27531); SU900;SU903;SU905;SU906;SU908;SU909. Feces of piglet

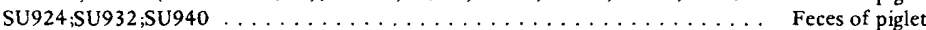

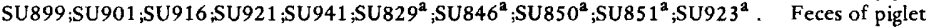

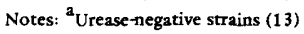

Bifidobacterium bifidum (Tissier) Orla-Jensen

E319f G. Reuter var. a (20); S28a (= ATCC 15696) C. Reuter var. b . . . . . . Feces of infant ATCC 11863 (212A) Lactobacillus bifidus subsp. pennsylvanicus György (7) . . Feces of infant

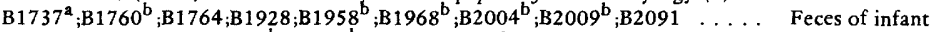
$\mathrm{B} 2093 ; \mathrm{B} 2193 ; \mathrm{B} 2475 ; \mathrm{B} 2531^{\mathrm{b}} ; \mathrm{B} 2554^{\mathrm{b}} ; \mathrm{B} 2614 ; \mathrm{B} 2658^{\mathrm{c}} ; \mathrm{B} 2660^{\mathrm{c}} ; \mathrm{B} 2662^{\mathrm{c}} \ldots \ldots$. Feces of infant

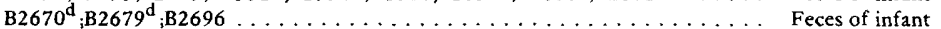

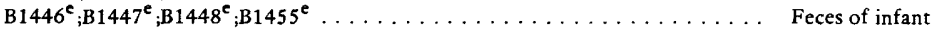
VT21;VT165;VT181;VT188;VT195/3;VT197/2;VT197/3;VT199 ...... Feces of calf VT261;VT286;VT320 . Notes: ${ }^{a}$ Ribose - ; ${ }^{b}$ melibioset; ${ }^{c}$ maltose and sucroset , fructose - (strain B2662

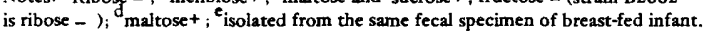

Bifidobacterium thermophilum Mitsuoka (Syn.: B. ruminale)

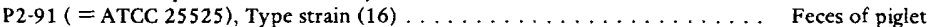
RU326 (= ATCC 25866), Type strain of B. ruminale (29) . . . . . . . . Rumen of cattle

Melezitose - and lactose - strains (Mitsuoka's biotype a (16)) RU294;RU349;RU366;RU368;RU402;RU433;RU439;RU647;RU673 . . . . . Rumen of cattle

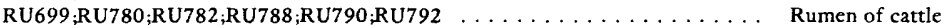

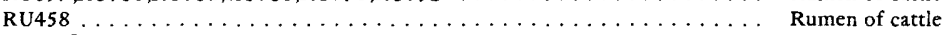

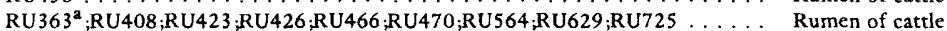

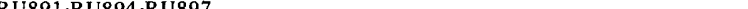

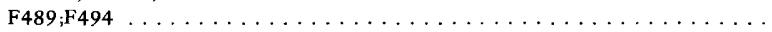
RU288;RU301;RU303;RU365;RU398;RU409;RU417;RU471;RU516;RU571

Melezitose - and lactose + strains (Mitsuoka's biotype $\mathrm{c}(16)$ ) $\mathrm{F} 50^{\mathrm{b}} ; \mathrm{F} 150^{\mathrm{b}} ; \mathrm{F} 334 ; \mathrm{F} 368 ; \mathrm{F} 438 ; \mathrm{F} 451 ; \mathrm{F} 452 ; \mathrm{F} 456 ; \mathrm{F} 491 ; \mathrm{F} 493^{\mathrm{c}} ; \mathrm{F} 499 ; \mathrm{F} 523 ; \mathrm{F} 524$. SU854;SU862;SU863 (see ref. 39) . . . . . . . . . . . . . . . . .

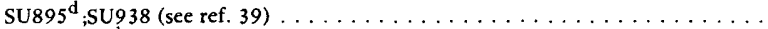

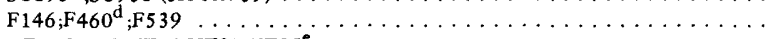
VT81;VT83;VT88;VT90;VT95e $\ldots \ldots \ldots \ldots \ldots \ldots \ldots \ldots$

Melezitose + and lactose + strains (Mitsuoka's biotype d (16))

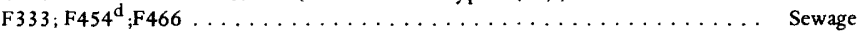
14-44;P16-6; NISSIN (Mitsuoka's biotype b, $c$ and $d$ resp.) . . . . . . . . Feces of pig

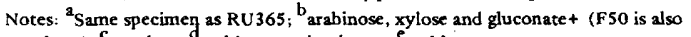

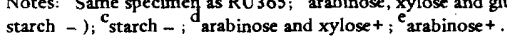

Bifidobacterium adolescentis Reuter

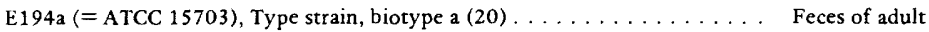

Mannitol + and sorbitol + (Reuter's biotype a (20))

$\mathrm{F}^{\mathrm{a}}{ }_{;} \mathrm{F}{ }^{\mathrm{a}}{ }_{;} \mathrm{Fq}^{\mathrm{a}}{ }_{;} \mathrm{F} 10 ; \mathrm{F}_{17}{ }^{\mathrm{a}} ; \mathrm{F} 21 ; \mathrm{F} 23 ; \mathrm{F} 147 ; \mathrm{F} 253 ; \mathrm{F} 268 \ldots \ldots \ldots \ldots$ Sewage

Mannitol+ and sorbitol - (Reuter's biotype b (20))

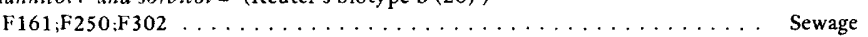

RU424;RU863;RU864;RU870 Group II (ref. 2) see also (30) . . . . . . . . . Rumen of cattle RU704 ${ }^{\text {ad }}$;RU724 ${ }^{\text {ad }}{ }_{; R}$ U822 ${ }^{\text {ad }}{ }_{i \text { RU716 }}{ }^{\text {ad }}$ Group III (ref. 2) $\ldots \ldots \ldots \ldots \ldots$. Rumen of cattle Mannitol - and sorbitol + ' (Reuter's biotype c (20))

$\mathrm{F} 2 ; \mathrm{F} 35^{\mathrm{a}} ; \mathrm{F4} 1 ; \mathrm{F} 80 ; \mathrm{F} 109^{\mathrm{c}} ; \mathrm{F} 110 ; \mathrm{F} 119 ; \mathrm{F} 123 ; \mathrm{F} 159 ; \mathrm{F} 160 ; \mathrm{F} 167 ; \mathrm{F} 200 ; \mathrm{F} 275^{\mathrm{a}} \ldots$ Sewage

$\mathrm{F} 283 ; \mathrm{F} 305 ; \mathrm{F} 318 ; \mathrm{F3} 38 ; \mathrm{F3} 31 ; \mathrm{F} 352 ; \mathrm{F3} 33 ; \mathrm{F365} ; \mathrm{F} 366 ; \mathrm{F377} ; \mathrm{F} 410 \ldots \ldots \ldots$ Sewage

Mannitol - and sorbitol - (Reuter's biotype d (20))

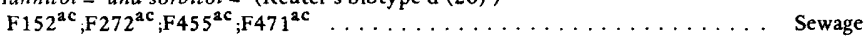

E305 (= ATCC 15704), E298b (=ATCC 15705), E319a $(=$ ATCC 15706)

(Reuter's biotypes $\mathrm{b}, \mathrm{c}$ and $\mathrm{d}$ respectively $(20)) \ldots \ldots \ldots \ldots \ldots \ldots$ Feces of adult

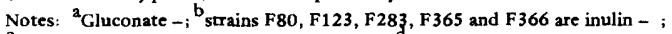

cellobiose - (F152,F455 are also salicin - ); arabinose and xylose -

Bifidobacterium indicum Scardovi and Trovatelli

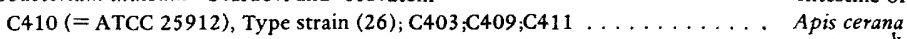

(Malaysia) $^{\mathbf{k}}$

$\mathrm{C} 418(=\mathrm{ATCC} 25913) \ldots \ldots \ldots \ldots \ldots \ldots \ldots \ldots \ldots \ldots$ Apis cerana

(Japan)

$\mathrm{C} 526, \mathrm{C} 557$

Apis cerana

(Philippines) $^{\mathrm{m}}$

$\mathrm{C} 528 ; \mathrm{C}^{2} 06^{2}$

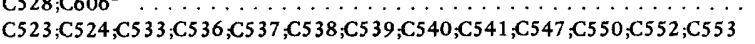

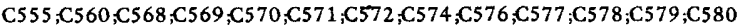

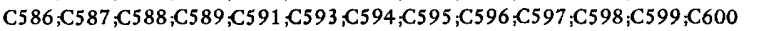
$\mathrm{C} 601, \mathrm{C} 603^{2}$ $\mathrm{C} 435 ; \mathrm{C} 436 ; \mathrm{C} 437 ; \mathrm{C} 440 ; \mathrm{C} 442 ; \mathrm{C} 443^{\mathrm{b}} ; \mathrm{C} 445 ; \mathrm{C} 447 ; \mathrm{C} 448 \ldots \ldots \ldots \ldots$

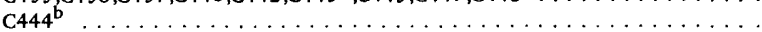

$\begin{array}{cll}\text { (Philippines) }^{\mathrm{m}} & & \\ ", & 9 & 8 \\ " & 9 & 6 \mathrm{a} \\ " & 9 & 6 \mathrm{a} \\ " & 9 & 6 \mathrm{a} \\ \text { (Philippines) }^{\mathrm{n}} & 9 & 6 \mathrm{a} \\ \text { " } & 7 & 8 \\ & 9 & 6 \mathrm{a}\end{array}$

$\begin{array}{ll}6 & 8 \\ 6 & 8 \\ 6 & 8 \\ 6 & 5\end{array}$

$\begin{array}{ll}7 & 7 \\ 7 & 7 \\ 7 & 7 \\ 7 & 7 \\ 7 & 7 \\ 7 & 8 \\ 7 & 7 \\ 7 & 7\end{array}$

$\begin{array}{ll}8 & 9 \\ 8 & 9 \\ 8 & \\ 8 & 9 \\ 8 & 9 \\ 8 & 9 a \\ 8 & 8 \\ 8 & 8 \\ 8 & 8 \\ & 7 \\ 8 & \\ 8 & 9 \\ 8 & 9 \\ 8 & 8 \\ 7 & 8 \\ & 7 \\ 8 & 9 \\ 8 & 8\end{array}$

8

5

5

5 
TABLE 1-continued

\begin{tabular}{|c|c|c|c|}
\hline \multirow[b]{2}{*}{ Strains } & \multirow[b]{2}{*}{ Source } & \multicolumn{2}{|c|}{ Electrophoretic form of } \\
\hline & & Transaldolase & 6PGD \\
\hline $\mathrm{C} 486^{\mathrm{i}}$ & $\begin{array}{l}\text { Apis dorsata } \\
\text { (Philippines) }^{\mathrm{m}}\end{array}$ & 9 & 6 \\
\hline 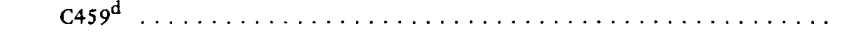 & $"$ & 9 & $6 a$ \\
\hline 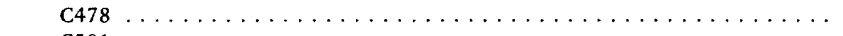 & $"$ & 8 & $9 \mathrm{a}$ \\
\hline 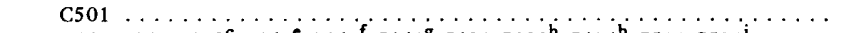 & $"$ & 8 & 8 \\
\hline $\mathrm{C} 449 ; \mathrm{C} 450 ; \mathrm{C} 453^{\mathrm{c}} ; \mathrm{C} 463^{\mathrm{e}} ; \mathrm{C} 465^{\mathrm{f}} ; \mathrm{C} 469^{\mathrm{g}} ; \mathrm{C} 481 ; \mathrm{C483}{ }^{\mathrm{h}} ; \mathrm{C} 484^{\mathrm{h}} ; \mathrm{C500} ; \mathrm{C} 501^{\mathrm{i}} \ldots \ldots$ & $"$ & 8 & 6 \\
\hline 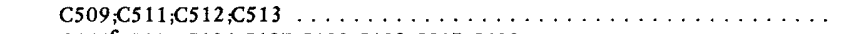 & $"$ & 8 & 6 \\
\hline 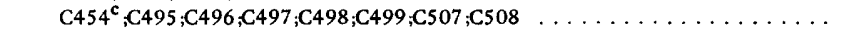 & $"$ & 7 & $9 \mathrm{a}$ \\
\hline 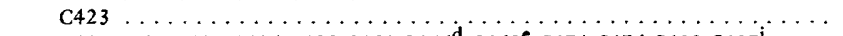 & $"$ & 7 & 9 \\
\hline $\mathrm{C} 421 ; \mathrm{C} 424 ; \mathrm{C} 425 ; \mathrm{C} 426 ; \mathrm{C} 428 ; \mathrm{C} 451 ; \mathrm{C} 456^{\mathrm{d}} ; \mathrm{C} 462^{\mathrm{e}} ; \mathrm{C} 474 ; \mathrm{C} 476 ; \mathrm{C} 482 ; \mathrm{C} 487^{\mathrm{i}} \ldots$ & $"$ & 7 & 8 \\
\hline 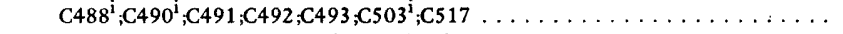 & $"$ & 7 & 8 \\
\hline 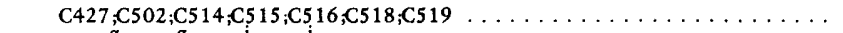 & $"$ & 7 & 7 \\
\hline 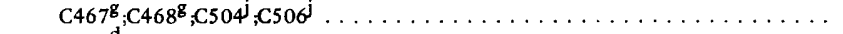 & $"$ & 7 & 6 \\
\hline 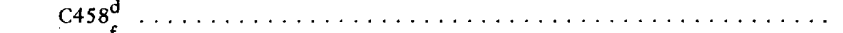 & $"$ & 7 & 6 \\
\hline 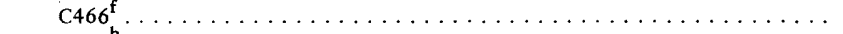 & $"$ & 6 & 8 \\
\hline 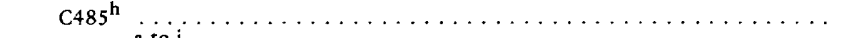 & $"$ & 6 & 6 \\
\hline \multicolumn{4}{|l|}{ 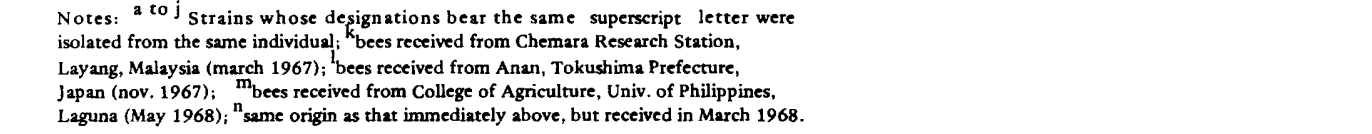 } \\
\hline \multicolumn{4}{|l|}{ Bifidobacterium asteroides Scardovi and Trovatelli } \\
\hline C51 $(=$ ATCC 25910$)$, Type strain $(26) \ldots \ldots \ldots \ldots \ldots \ldots \ldots \ldots \ldots \ldots \ldots \ldots \ldots$ & $\begin{array}{l}\text { Apis mellifera } \\
\text { (Europe) }^{\mathrm{a}}\end{array}$ & 8 & $10 \mathrm{a}$ \\
\hline 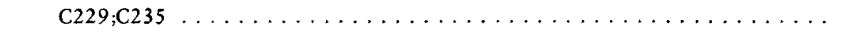 & $"$ & $9 \mathrm{a}$ & $10 \mathrm{a}$ \\
\hline 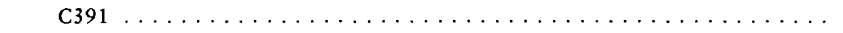 & $"$ & 9 & 13 \\
\hline 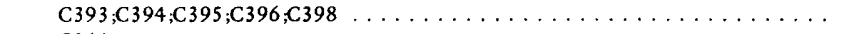 & $"$ & 9 & 12 \\
\hline 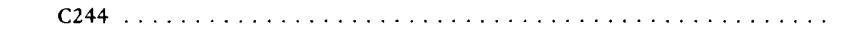 & $"$ & 9 & $10 b$ \\
\hline 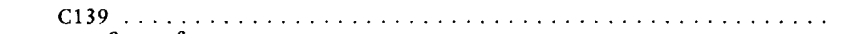 & $"$ & 9 & $10 \mathrm{a}$ \\
\hline 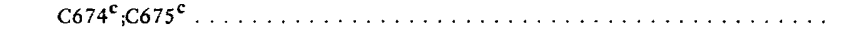 & (Philippines) $^{\mathrm{b}}$ & 9 & $10 a$ \\
\hline 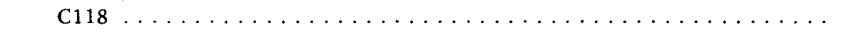 & $(\text { Europe })^{\mathrm{a}}$ & $8 \mathrm{~b}$ & 13 \\
\hline 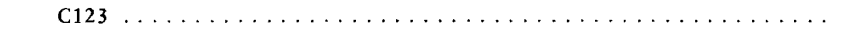 & $"$ & $8 \mathrm{~b}$ & $10 \mathrm{~b}$ \\
\hline 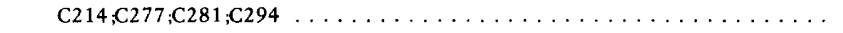 & $"$ & $8 \mathrm{~b}$ & $10 \mathrm{a}$ \\
\hline 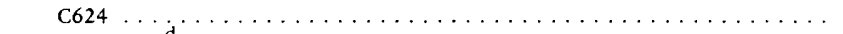 & (Philippines) $^{\mathrm{b}}$ & $8 \mathrm{~b}$ & $10 \mathrm{a}$ \\
\hline 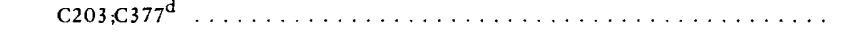 & ${\text { (Europe })^{2}}^{2}$ & $8 \mathrm{a}$ & $10 b$ \\
\hline 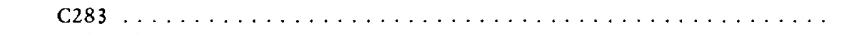 & $" \quad$ s & $8 \mathrm{a}$ & $10 \mathrm{a}$ \\
\hline 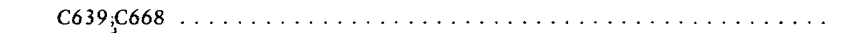 & (Philippines) $^{\mathrm{b}}$ & $8 \mathrm{a}$ & $10 \mathrm{a}$ \\
\hline 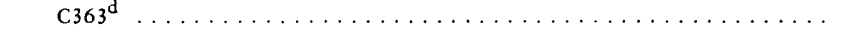 & $(\text { Europe })^{2}$ & $8 \mathrm{a}$ & $9 \mathrm{a}$ \\
\hline 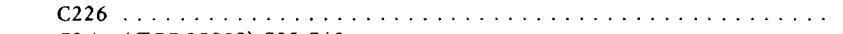 & $"$ & 8 & 12 \\
\hline 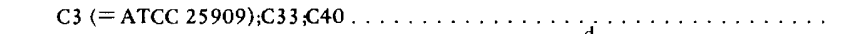 & $"$ & 8 & 11 \\
\hline $\mathrm{C} 71 ; \mathrm{C} 73, \mathrm{C} 74, \mathrm{C} 184, \mathrm{C} 197, \mathrm{C} 250, \mathrm{C} 301, \mathrm{C} 338 ; \mathrm{C} 350 ; \mathrm{C} 375^{\mathrm{d}} ; \mathrm{C} 387 \ldots \ldots$ & $"$ & 8 & $10 \mathrm{~b}$ \\
\hline 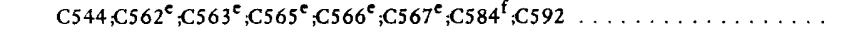 & Apis ceranab & 8 & $10 \mathrm{~b}$ \\
\hline 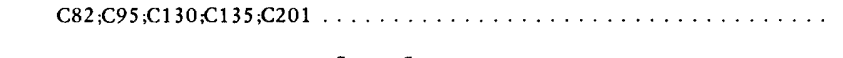 & $\begin{array}{l}\text { Apis mellifera } \\
\text { (Europe) }^{\mathbf{2}}\end{array}$ & 8 & $10 \mathbf{a}$ \\
\hline 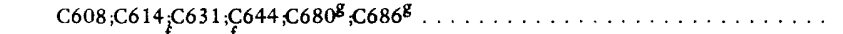 & $"$ p & 8 & $10 \mathrm{a}$ \\
\hline 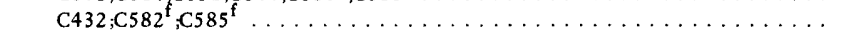 & Apis cerana ${ }^{\mathrm{b}}$ & 8 & $10 \mathrm{a}$ \\
\hline 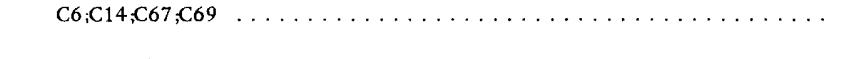 & $\begin{array}{l}\text { Apis mellifera } \\
\text { (Europe) }\end{array}$ & 8 & 10 \\
\hline 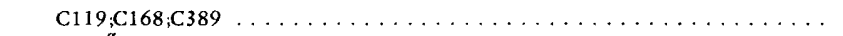 & " & 8 & $9 \mathrm{~b}$ \\
\hline 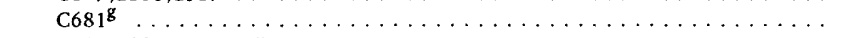 & (Philippines) $^{\mathrm{b}}$ & 8 & $9 \mathrm{~b}$ \\
\hline 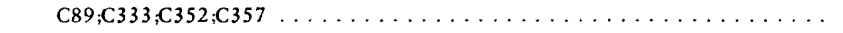 & 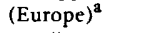 & 8 & $9 \mathrm{a}$ \\
\hline 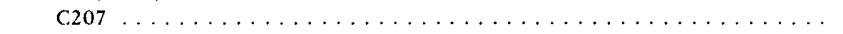 & " & 8 & 9 \\
\hline 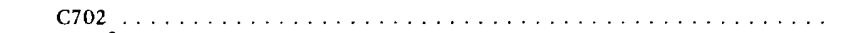 & (Philippines) $^{\mathbf{b}}$ & $7 \mathbf{a}$ & $10 \mathrm{~b}$ \\
\hline 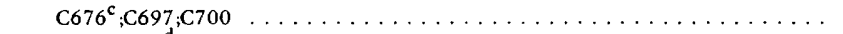 & & $7 \mathrm{a}$ & $10 \mathrm{a}$ \\
\hline 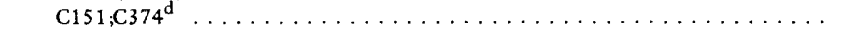 & (Europe) $^{\mathbf{a}}$ & 7 & $10 \mathrm{a}$ \\
\hline \multirow{2}{*}{\multicolumn{4}{|c|}{$\begin{array}{l}\text { Notes: }{ }^{a} \text { Countries of origin are reported in ref. 26; }{ }^{b} \text { bees received from College } \\
\text { of Agriculture, Univ. of Philippines, Laguna (June-Nov. 1968); to }{ }_{\text {same }} \\
\text { superscript letter indicates isolation from the same intestine. }\end{array}$}} \\
\hline & & & \\
\hline \multicolumn{4}{|l|}{ "Minimum" DNA humology group (27) } \\
\hline F392 $(=$ ATCC 27538), Reference strain (27); F399 $\ldots \ldots \ldots \ldots \ldots$ & Sewage & 10 & 6 \\
\hline
\end{tabular}

or smeared bands, or one or two more faint anodic bands subordinate to the major one, expecially when concentrated extracts were used; appropriate dilution of the extracts obviated this effect. The occurrence of such sub- bands, observed only with 6PGD staining, was by no means repeatable from run to run; hence, at this point it can only be assumed that there is only one band per enzyme per strain.

Whereas transaldolase staining was invariably 


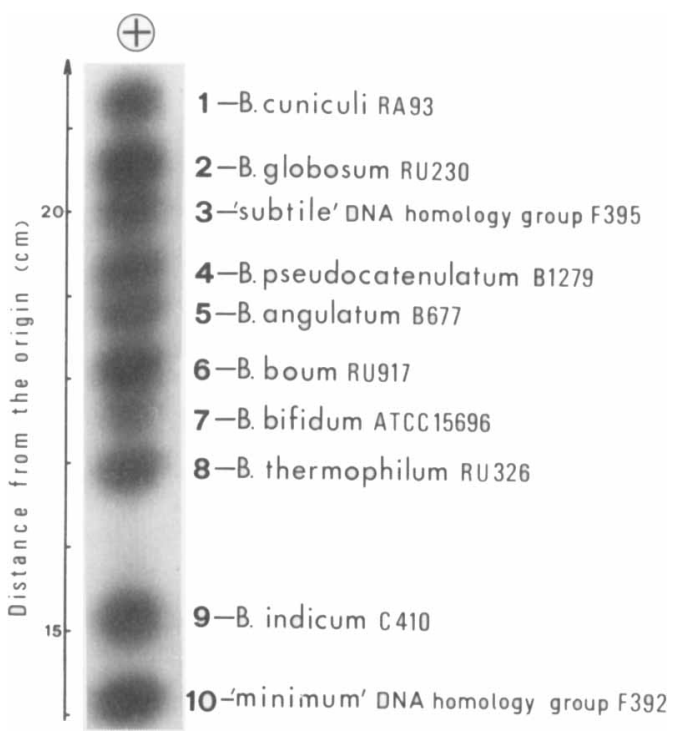

Fig. 1. Coelectrophoretic run of pooled cell-free extracts from 10 reference Bifidobacterium strains after staining for transaldolase.

positive even with very dilute extracts (generally 1:10 for this staining), the staining for 6PGD failed with strains of $B$. pullorum, some strains of $B$. longum, and most $B$. dentium strains. The absence, or better the indeterminability, of this enzyme in B. pullorum strains has already been shown (37), but the low content of this enzyme in $B$. dentium strains was rather surprising because of the ability of this species to develop with gluconate as a fermentable substrate (22). Trials were made to determine the sensitivity of the staining procedure for 6PGD under our conditions of electrophoresis; $0.05 \mathrm{mU}$ of enzyme appeared to be the lowest quantity for a positive staining after 3 to $4 \mathrm{~h}$ of storage in the reaction mixture at $37^{\circ} \mathrm{C}$. This means that extracts that were not stainable for $6 \mathrm{PGD}$, which have a protein content of 10 to $20 \mathrm{mg} / \mathrm{ml}$, do not possess a dehydrogenase specific activity higher than 1 $\times 10^{-4}$ to $2 \times 10^{-4} \mathrm{U}$ (used in $20-\mu \mathrm{l}$ samples per slot). The $B$. dentium strains, which were positive for 6PGD staining (see below), exhibited specific activities in the range from 0.005 to 0.01 $\mathrm{U}$, i.e., from 50 - to 100 -fold the minimal stainable level of the enzyme. 6PGD was invariably oxidized nicotinamide adenine dinucleotide phosphate dependent.

Distribution of isozymes. The isozymes of transaldolase and 6PGD found among the strains studied are presented in Tables 1 and 2. In Table 1 the results are reported in detail, and additional information is given concerning the sources and some distinguishing fermentative characters of the strains studied. In Table 2 are reported: (i) frequencies of the zymographic patterns, (ii) the bands which may be of significance as species markers, (iii) matchings between isozymes in different species, and (iv) indications as of distinguishing between the species by means of 3-phosphoglyceraldehyde dehydrogenase staining. The results of this study are graphically presented in Fig. 3; in these diagrams the species-specific zymograms are reported together with gross indications of the frequencies of the enzymic bands found in each species.

The high degree of isozyme variability displayed by $B$. asteroides and $B$. indicum is illustrated in the isozyme distribution histograms given in Fig. 4.

\section{DISCUSSION}

The eventual taxonomic and ecological significance of electrophoretic data of the kind reported in this study obviously depends on the number of strains of a given species studied and on the variety of habitats from which the strains were isolated.

$B$. globosum Scardovi et al. (29) is considered by Rogosa (21) to be identical with $B$. pseudolongum Mitsuoka (16). However, we still maintain that these species are unique due to differences in the guanine plus cytosine contents of their DNA (some 3\%) and to their DNA homol-

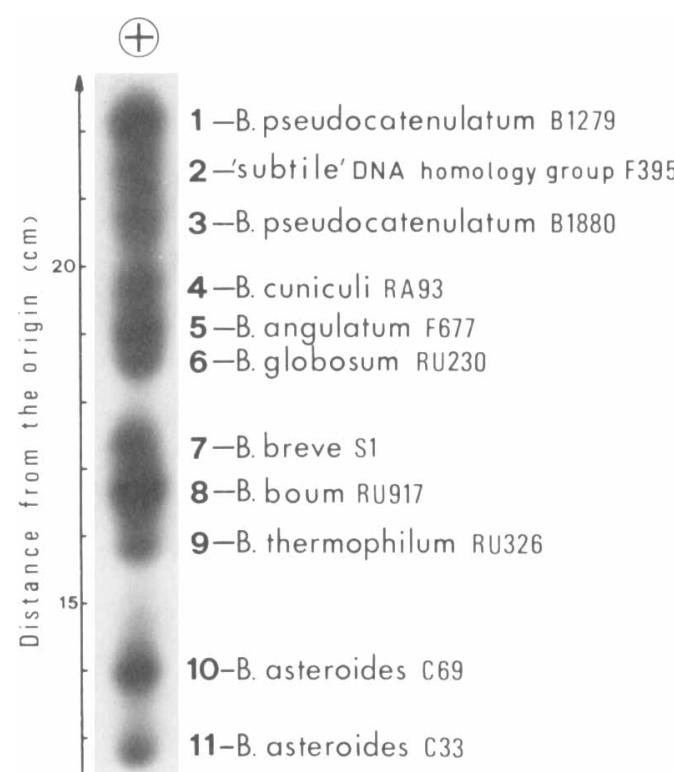

Fig. 2. Coelectrophoretic run of pooled cell-free extracts from 11 Bifidobacterium reference strains after staining for $6 P G D$. 

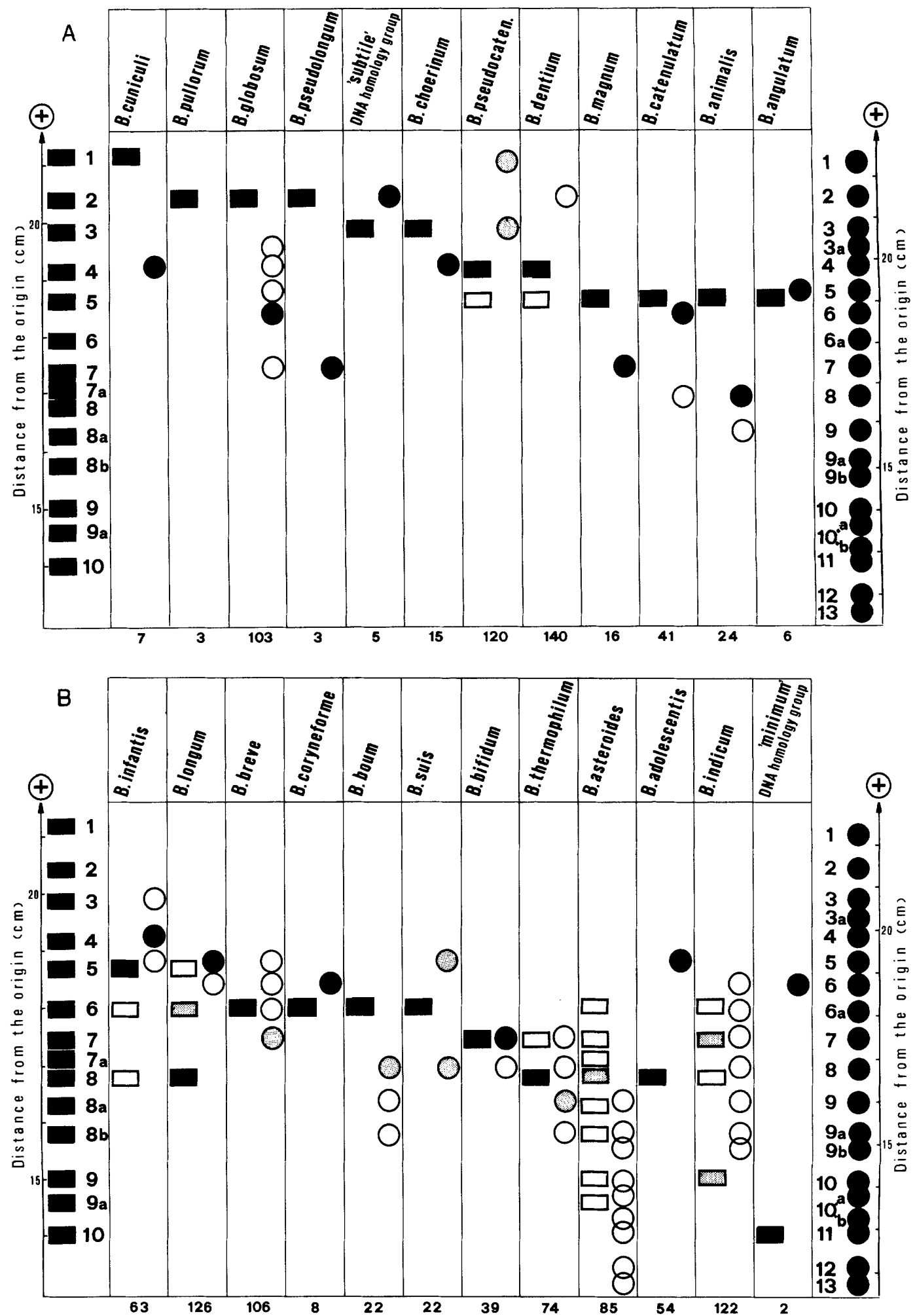

FIG. 3. Diagrammatic representations of the electrophoretic forms of transaldolase and 6PGD found in the genus Bifidobacterium. The numbers on the sides of the diagrams refer to the isozymes of transaldolase (squares) and 6PGD (circles). Symbols: $\square$ and 9 , isozymes found in 75 to $100 \%$ of strains; 10 and $\odot$, isozymes found in 40 to $60 \%$ of strains; $\square$ and $\bigcirc$, isozymes found in less than $30 \%$ of strains. The numbers at the bottom of each column refer to the number of strains studied. 
TABLE 2. Transaldolase and 6FGD isozyme frequencies in the Bifidobacterium strains studied

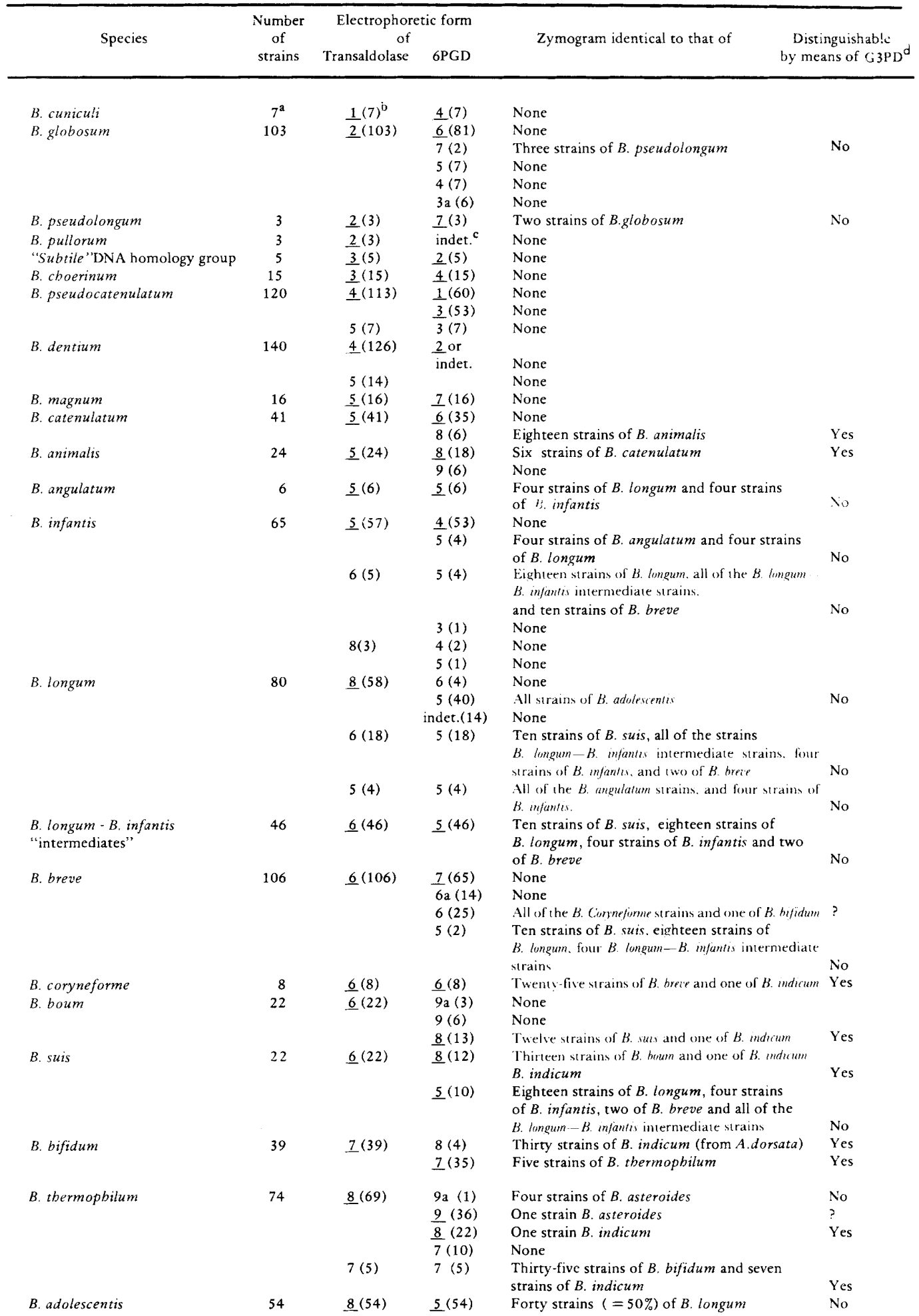


TABLE 2-continued

\begin{tabular}{|c|c|c|c|c|c|}
\hline Species & $\begin{array}{c}\text { Number } \\
\text { of } \\
\text { strains }\end{array}$ & $\begin{array}{r}\text { Electrophore } \\
\text { of } \\
\text { Transaldolase }\end{array}$ & Electrophoretic form & Zymogram identical to that of & $\begin{array}{l}\text { Distinguishable } \\
\text { by means of G3PD }\end{array}$ \\
\hline \multirow[t]{23}{*}{ B. asteroides } & 85 & $9 \mathrm{a}(2)$ & $10 \mathrm{a}(2)$ & None & \\
\hline & & $9(10)$ & $13(1)$ & None & \\
\hline & & & $12(5)$ & None & \\
\hline & & & $10 \mathrm{~b}(1)$ & None & \\
\hline & & & $10 \mathrm{a}(3)$ & None & \\
\hline & & $8 b(7)$ & $13(1)$ & None & \\
\hline & & & $10 b(1)$ & None & \\
\hline & & & $10 \mathrm{a}(5)$ & None & \\
\hline & & $8 \mathrm{a}(6)$ & $10 \mathrm{~b}(2)$ & None & \\
\hline & & & $10 \mathrm{a}(3)$ & None & \\
\hline & & & $9 \mathrm{a}(1)$ & None & \\
\hline & & $\underline{8}(51)$ & $12(1)$ & None & \\
\hline & & & $11(3)$ & None & \\
\hline & & & $10 \mathrm{~b}(19)$ & None & \\
\hline & & & $10 a(15)$ & None & \\
\hline & & & $10(4)$ & None & \\
\hline & & & $9 b(4)$ & None & \\
\hline & & & $9 a(4)$ & $\begin{array}{l}\text { One strain } B \text {. thermophitum and one strain of } B \text {. } \\
\text { mdicum }\end{array}$ & No \\
\hline & & & $9(1)$ & Thirty-six strains of B.thermophilum & $?$ \\
\hline & & $7 \mathrm{a}(4)$ & $10 b(1)$ & None & \\
\hline & & & $10 a(3)$ & None & \\
\hline & & $7(2)$ & $10 a(2)$ & None & \\
\hline & & $6(3)$ & $10 \mathrm{~b}(3)$ & None & \\
\hline \multirow[t]{14}{*}{ B. indicum } & 122 & $9(53)$ & $9 \mathrm{~b}(2)$ & None & \\
\hline & & & $9(5)$ & None & \\
\hline & & & $8(2)$ & None & \\
\hline & & & $6(1)$ & None & \\
\hline & & & $6 a(43)$ & None & \\
\hline & & $8(17)$ & $9 \mathrm{a}(1)$ & $\begin{array}{l}\text { Four strains of } B \text {. asterstdes and one strain of } B \text {. } \\
\text { themophlum }\end{array}$ & No \\
\hline & & & $8(1)$ & Twenty-two strains of $B$. thermophilum & Yes \\
\hline & & & $6(15)$ & None & \\
\hline & & $7(50)$ & $9 \mathrm{a}(8)$ & None & \\
\hline & & & $8(30)$ & Four strains of $B$. bifidum & Yes \\
\hline & & & $7(7)$ & $\begin{array}{l}\text { Thirty-five strains of } B \text {. bifidum and five } \\
\text { strains of } B \text {. thermophilum }\end{array}$ & Yes \\
\hline & & & $6(5)$ & None & \\
\hline & & $6(2)$ & $8(1)$ & $\begin{array}{l}\text { Twelve strains of } B \text {. suis and thirteen } \\
\text { strains of } B . \text { boum }\end{array}$ & ? \\
\hline & & & $6(1)$ & $\begin{array}{l}\text { Titentr-fint strams of } B \text { breir and all of the strains of } \\
\text { B. corneforme }\end{array}$ & ? \\
\hline "Minimum" DNA homology group & 2 & $\underline{10}(2)$ & $\underline{6}(2)$ & None & ? \\
\hline
\end{tabular}

a The underlined numbers refer to the most commonly found isozymes in each species (species-specific markers).

${ }^{b}$ Numbers in parentheses are numbers of strains displaying the same isozyme.

' indet., Indeterminable with spot staining (see under Results).

${ }^{d}$ G3PD, 3-Phosphoglyceraldehyde dehydrogenase.

ogy level (near $70 \%$ ). The similarity in their zymogram patterns seems to confirm Rogosa's opinion, but the strains of $B$. pseudolongum included in this study are too few to give significant results. All of the strains of $B$. globosum share the transaldolase isozyme 2 but display at least five 6PGD isozymes, the most frequent of which, number 6 , is shared by all of the strains isolated from rumen and sewage and from the feces of piglets, calves, lambs, and human infants. The other isozymes are found, although less frequently than isozyme 6 , in the strains isolated from rat, chicken, and rabbit feces. It should be mentioned that $B$. globosum is the only species found in such a large variety of animal intestinal habitats. The variability of 6PGD seems to be in some way habitat dependent. In the original description of $B$. globosum, this organism is reported as never fermenting xylose, mannitol, or gluconate and only rarely fermenting arabinose (29). The pentoses, mannitol, and gluconate were fermented by the strains of $B$. globosum isolated by Crociani et al. (2). Other biovars were found by Trovatelli and 


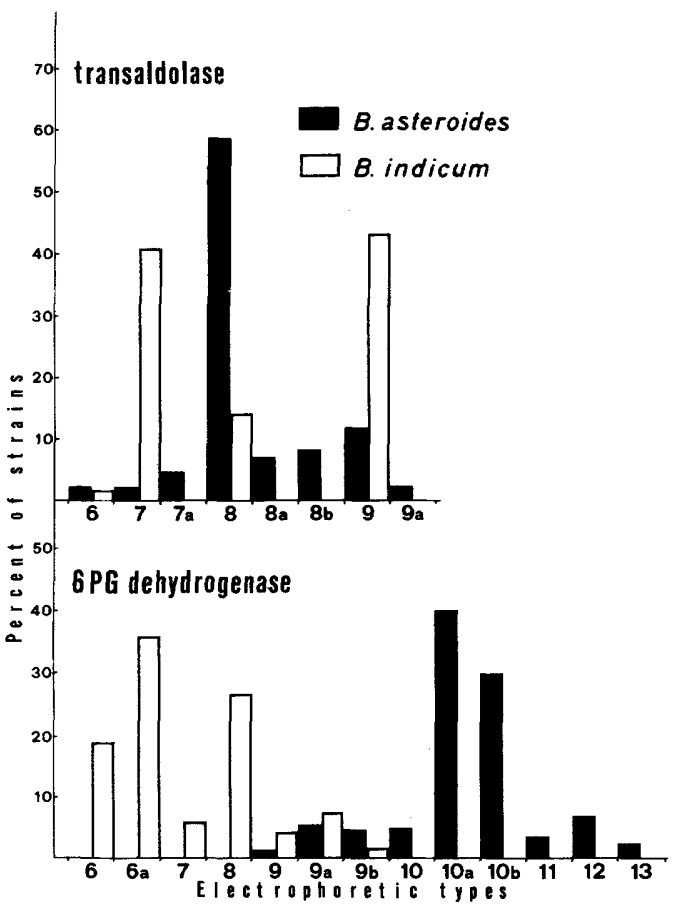

Fig. 4. Frequency histograms of the transaldolase and $6 P G D$ electrophoretic forms of strains of $B$. as. teroides and $B$. indicum. Numbers on the abscissa correspond to those reported in Fig. 3.

Matteuzzi (38) and by Zani et al. (39). Most of the $B$. globosum strains studied here fermented arabinose and xylose, as did strains of $B$. pseudolongum. No particular isozyme pattern was found to correlate with these biovars. It should be mentioned that $B$. globosum biovars that ferment arabinose and xylose and $B$. pseudolongum cannot be distinguished from $B$. angulatum on the basis of sugars fermented; their distinction should be based on the guanine plus cytosine content of their DNA, DNA homology, and morphology (22). The differences in their electropherograms (see Table 2) could be of diagnostic value.

Transaldolase isozyme 4 was found in $94 \%$ of the strains of B. pseudocatenulatum and in $90 \%$ of the strains of $B$. dentium, whereas the less mobile band 5 , found in a minority of the strains of both species, was found in all of the strains of $B$. magnum, $B$. catenulatum, $B$. animalis, and $B$. angulatum and in most of the strains of $B$. infantis (see Table 2). Among these species, $B$. pseudocatenulatum and $B$. catenulatum are the most closely related genetically: their DNA homology is in the range of 60 to $80 \%$ (28). Differences in the structure of their cells wall peptidoglycan, in the guanine plus cytosine content of their DNA, and in their fermentation of starch were distinctive traits which correlated with the difference in their DNA base sequences. The zymograms of these two species are quite different, especially with respect to the mobilities of their 6PGDs (Table 2); this adds support to their taxonomic distinction. It should be mentioned that among the 64 strains of $B$. pseudocatenulatum studied, the following 11 were included: six strains (B2247 to B2252, inclusive) from the feces of a breast-fed infant, and two (VT74 and VT77) from the feces of suckling calf. These strains, phenotypically members of $B$. pseudocatenulatum and with zymograms the same as that of $B$. pseudocatenulatum (pattern 4-3), behaved as "intermediates" between $B$. pseudocatenulatum and $B$. catenulatum with regard to DNA-DNA hybridization (ref. 28, Table 6). Furthermore, the strains of $B$. pseudocatenulatum isolated from suckling calves have 6PGD isozyme 3, whereas most of those from the feces of infants have the more anodic isozyme 1 (see Table 1).

Although identical electrophoretic mobilities of enzymes do not necessarily mean identical primary structures, the strong similarity of the electrophoretic patterns of $B$. pseudocatenulatum and $B$. dentium (see Table 2) is somewhat surprising. The two species are less than $20 \%$ related (DNA homology), and they diverge in a number of characters like guanine plus cytosine content of DNA, cell wall peptidoglycan structure, sugars fermented, and habitat $(22,28) . B$. dentium, the unique bifidobacterium found constantly in human caries (unpublished results), has also been found in the oral cavity and appendix and in abscesses of humans (see below); its presence in the feces of human infants and of rabbits is very rare (unpublished data). On the other hand, B. pseudocatenulatum is common in the feces of human infants, in suckling calves, and in sewage (28). The presence of 6PGD isozyme 2 seems to be distinctive for $B$. dentium cells grown on gluconate; cells grown on glucose, with the exception of those of strains isolated from the feces of human infants and from abscesses in humans (Actinomyces eriksonii), do not possess stainable levels of this enzyme (see Materials and Methods). The two strains of $A$. eriksonii (ATCC 15423 and ATCC 15424), isolated by Georg et al. (5) and classified as Bifidobacterium eriksonii by Holdeman and Moore $(8)$, produced the same zymogram pattern as $B$. dentium (pattern 4-2; see Table 1). This is in accord with the high DNA homology (80 to $100 \%$; Scardovi, unpublished data) we have found between these two strains and the type strain of B. dentium (B764 = ATCC 27534) used as reference. Recently Mitsuoka et al. (17) pro- 
posed that the name $B$. eriksonii is a synonym of $B$. adolescentis on the basis of their phenotypic identity. Actually, B. dentium is indistinguishable from $B$. adolescentis on phenotypic grounds; at present, only DNA homology and zymograms are helpful in distinguishing between these organisms (see Table 2).

Preliminary data on the DNA homology relationships between $B$. infantis Reuter (syn.: $B$. liberorum and $B$. lactentis) and $B$. longum Reuter, obtained with Reuter's strains and a few additional isolates from the feces of breast-fed infants, indicated considerable interspecies relatedness (32). A large number of strains isolated in our laboratory from the feces of either breastor bottle-fed infants was subsequently studied by means of DNA-DNA hybridization, and the interspecific levels of DNA homology between the two species were found to vary in the range of 65 to $80 \%$. However, as many as 180 strains isolated successively from the feces of suckling calves could not be identified with either species by means of this technique: they were over $80 \%$ related to the reference strains of both species. Conversely, selected strains of $B$. infantis and B. longum displayed nearly identical high degrees of relatedness ( 75 to $80 \%$ ) to selected reference strains from calves (unpublished data). The strains from calves are referred to here as intermediates between $B$. infantis and $B$. longum; their existence could place some doubt on the advisability of recognizing $B$. infantis and $B$. longum as separate species and would seem to support the idea that these two species actually form a "continuum" where the central position could well be occupied by the strains from calf feces. In any event, sugar fermentation patterns are largely shared by the $B$. infantis and $B$. longum homology groups. Arabinose and xylose, which according to Reuter (20) are not fermented by $B$. infantis, are fermented by most of our strains of $B$. infantis, which are all melezitose negative, as was reported by Reuter for his strains. Melezitose-positive and -negative strains were found in the $B$. longum group as well as ribose-positive and -negative strains. All of the calf strains fermented arabinose and xylose; melezitose and ribose were variably fermented (Table 1; also, unpublished data). No wonder, therefore, that the transaldolase and 6PGD zymograms overlap extensively in these bifidobacteria (see Tables 1 and 2 and Fig. 3B). Although $92 \%$ of the $B$. infantis strains displayed transaldolase 5 and 6 PGD band 4 , a pattern not shared by any other bifidobacteria (the other species that have transaldolase 5 have less anodic 6PGD bands), only $72 \%$ of the $B$. longum strains have the less anodic transaldolase isozyme 8 . The transaldolase isozyme 6 of intermediate mobility is common to all of the strains from calves and to 8 and $20 \%$ of the $B$. infantis and $B$. longum strains, respectively.

All $B$. breve strains, which are commonly found in the feces of human infants, in human vagina, and in the feces of suckling calves, have a form of transaldolase (isozyme 6; see Tables 1 and 2 and Fig. 3B) that is apparently identical with that of the $B$. infantis- $B$. longum intermediates from calves. This is understandable: recent investigations (unpublished data) have shown that $B$. breve is the species most closely related to either $B$. infantis or $B$. longum (50 to $75 \%$ DNA homology). Rather unexpectedly, $B$. coryneforme, $B$. boum, and $B$. suis possess the same transaldolase (isozyme 6), although these species differ from $B$. breve in a number of phenotypic traits and in habitat (see Table 1) and are genetically unrelated to $B$. breve $(14,28$, $30)$. This similarity is merely superficial, however; the transaldolases of $B$. breve, $B$. infantis, and $B$. longum are immunologically different from those found in the other species mentioned (34). The transaldolases of the $B$. breve strains that fermented arabinose and xylose and those of the strains from the human vagina and the feces of suckling calves were not electrophoretically distinct from those of the strains isolated from the feces of human infants (see Table 1).

A new species, $B$. boum, has recently been described (28). This species was found in the rumen contents of cattle, which is also the habitat of $B$. thermophilum Mitsuoka (syn [21]: $B$. ruminale Scardovi et al.). The two species are separated primarily on DNA homology (60 to $70 \%$ relatedness) and on differences in the structures of their cell wall peptidoglycans (28). The difference in the electrophoretic mobilities of their specific transaldolases adds support to the taxonomic separation of these species; the electrophoretic forms of their 6PGDs are identical (Tables 1 and 2). Of the $B$. thermophilum strains, five were isolated from the feces (two specimens) of suckling calves. These strains can be distinguished from those isolated from the rumen of cattle, from sewage and from the feces of pigs because they have transaldolase isozyme 7 (Table 1), an isozyme with a mobility intermediate between that (isozyme 8 ) of the vast majority of $B$. thermophilum strains and that (isozyme 6) of the genetically related $B$. boum. These findings are similar to those obtained with the $B$. infantis- $B$. longum intermediate strains isolated from the same habitat: these strains possessed a transaldolase band with a mobility intermediate between those of $B$. infantis and $B$. longum. In the first case, however, the strains (VT81, VT83, VT88, VT90, and VT95) were undoubtedly memijers of $B$. thermophilum on 
the basis of DNA-DNA hybridization results (unpublished data), whereas in the latter case the strains were genetically intermediate between $B$. infantis and $B$. longum (see above). These findings have possible ecological significance because they seem to indicate that the bifidobacteria found in the feces of suckling animals are in some way different from those found in the rumen contents of the same animal, even though these organisms belong to the same genospecies.

$B$. bifidum is characterized by the zymogram pattern 7-7; 6PGD band 8 was found in four strains only (Tables 1 and 2). The 7-7 pattern is shared by the few strains of $B$. thermophilum isolated from the feces of calves (see above), whereas the pattern $7-8$ is displayed by a large number of $B$. indicum strains isolated from $A$ pis dorsata (Table 2). In any event, B. bifidum strains can be distinguished because they possess a more anodic 3-phosphoglyceraldehyde dehydrogenase. $B$. bifidum strains isolated from the feces of calves and of breast-fed infants have the same transaldolase and 6PGD electrophoretic pattern (Table 1). These two habitats, therefore, harbor not only the same Bifidobacterium species-viz. B. bifidum, B. pseudocatenulatum, $B$. infantis, $B$. longum, and $B$. brevebut also identical enzymatic biovars of these species.

The 54 strains of $B$. adolescentis investigated in this study displayed zymogram pattern 8-5 (Table 2) even though they were of different origins, viz. human feces, sewage, and rumen of cattle (Table 1), and possessed different fermentation patterns (Reuter's biovars [20] and pentose-negative strains from rumen [2]). Because we had only four strains from the feces of adult humans (Reuter's strains, see Table 1), a habitat which has been reported to be the one preferred by this species $(16,20)$, our data might not be representative of this species. The $B$. adolescen$t i s$ strains studied could not be distinguished on the basis of zymogram pattern from nearly half of the $B$. longum strains isolated from the feces of infants and included in this study. Furthermore, these two groups of strains possess 3-phosphoglyceraldehyde dehydrogenases with identical electrophoretic mobilities (unpublished data). Preliminary immunological data on the transaldolases of the bifidobacteria (34) seem to confirm this apparent relatedness: the transaldolase of $B$. adolescentis is immunologically more related to that of $B$. longum than to those of $B$. catenulatum, $B$. dentium, $B$. angulatum, and $B$. pseudocatenulatum. However, B. adolescentis is apparently more related phenotypically and genetically (DNA homology) to these species than to $B$. longum (unpublished data).
It is evident that the DNA relatedness between $B$. adolescentis and $B$. longum, determined so far only with a limited number of isolates (32), needs to be more precisely defined, especially since the taxonomic status of $B$. longum seems to be unclear (see above).

$B$. asteroides and $B$. indicum have been isolated from the intestines of Apis mellifera $\mathrm{L}$. (mostly of the race ligustica, but also mellifera and caucasica), Apis cerana Fabr. (A. indica), and Apis dorsata Fabr. The specimens of $A$. mellifera were obtained from various localities in Italy as well as from Greece, France, Germany, Norway, the Soviet Union, the Philippines, and other countries (details in ref. 26). The origins of $A$. cerana and $A$. dorsata are given in Table 1 . B. asteroides and $B$. indicum are highly variable with respect to the transaldolases and 6PGDs they produce (see Tables 1 and 2 and Fig. 4). As many as eight transaldolases and nine 6PGDs were detected in $B$. asteroides (85 strains), and four and seven, respectively, in $B$. indicum (124 strains). Here again, however, each strain exhibited only one band for each allozyme. Transaldolase zymograms are of little value in differentiating the two species because of the large number of band matchings; on the other hand, limited overlapping occurs with the 6PGD isozymes (see Fig. 4 and 5). $B$. asteroides is unique among the bifidobacteria in that it possesses the less mobile variants of 6PGD (Fig. 3B). The differences in the morphology, nutrition, and structure of cell wall peptidoglycan (11) of $B$. asteroides and $B$. indicum are such as to warrant their separation into different taxa (26). However, their reciprocal DNA homology (some 30\%; see ref. 32) is indicative of a certain relatedness because little or no homology was demonstrated with all other bifidobacteria used as references. Admittedly, only a few interspecific homology experiments were performed (32); additional experiments now under way will probably reveal higher levels of interspecific DNA relatedness.

The frequency distribution of transaldolase isozymes (Fig. 5) and the data summarized in Table 2 reveal some interesting facts. The distribution of $B$. indicum transaldolases is asymmetrical, with two isozymes (viz. 7 and 9) displaying nearly the same high frequency. Isozyme 7 is found almost exclusively in the strains isolated from Apis dorsata, whereas the less anodic isozyme 9 is found in the strains from Apis cerana ( $A$. indica) (see Table 1). Extensive investigations are needed to determine whether the bifidobacteria from $A$. cerana can be distinguished from those from $A$. dorsata, two species of asiatic bees of which specimens were received from Malaysia, Japan, and the Philippines, on 
the basis of DNA-DNA hybridization. The few data reported elsewhere (32), obtained with the DNA of strain C410 (=ATCC 25912) isolated from $A$. cerana, are suggestive that this can be achieved. The frequency distribution of isozymes is by far more symmetrical in $B$. asteroides, and there is no indication, according to experiments now under way, that groups of isolates of this species can be separated by means of DNA-DNA hybridization. As many as 23 different combinations of electrophoretic types of transaldolase and 6PGD are listed for $B$. asteroides in Table 2. If one considers that not less than six electrophoretic forms of 3-phosphoglyceraldehyde dehydrogenase have been preliminarily recognized among these strains (unpublished data), one could probably characterize each $B$. asteroides isolate by a strainspecific pattern of these three enzymes. It is not known, obviously, whether this high degree of variability is intrinsic to the genetic structure of the organism itself or is related to the rapidly changing environment the organism meets as the physiology of the host changes during its short life (see ref. 9, 15, 18, 19).

The bifidobacterial transaldolase of least electrophoretic mobility was found in the two strains isolated from sewage and assigned to the "minimum" DNA homology group (27). However, their 6PGD isozyme is apparently more anodic than expected (Fig. 3B). This observation, which at present is only anecdotal, stems from a cursory comparison of the relative positions of the isozymes graphically presented in Fig. 3; the decrease in the electrophoretic mobility of the transaldolases from $B$. cuniculi to $B$. asteroides is seemingly accompanied by a decrease in the mobility of their 6PGD isozymes. Electrophoretic studies on other enzymes are needed for elucidating this point, which might have some evolutionary significance.

\section{REPRINT REQUESTS}

Address reprint requests to: Dr. Vittorio Scardovi, Istituto di Microbiologia Agraria, Università di Bologna, Via Filippo Re 6, Bologna, Italy.

\section{LITERATURE CITED}

1. Brewer, G. T. 1970 . An introduction to isozyme techniques. Academic Press, New York.

2. Crociani, F., V. Scardovi, and L. D. Trovatelli. 1970. Mannitol fermenting bifids from rumen and their DNA homology relationships. Ann. Microbiol. 20:101-107.

3. DeMann, J. C., M. Rogosa, and E. M. Sharpe. 1960. A medium for the cultivation of lactobacilli, J. Appl. Bacteriol. 23:130-135.

4. DeVries, W., S. J. Gerbrandy, and A. H. Stouthamer. 1967. Carbohydrate metabolism in Bifidobacterium bi. fidum. Biochim. Biophys. Acta 136:415-425.

5. Georg, L. K., G. W. Robertstad, S. A. Brinkman, and M. D. Hicklin. 1965. A new pathogenic anaerobic Actinomyces species. J. Infect. Dis. 115:88-99.
6. Gillespie, J. H., and K. Kojima. 1968. The degree of polymorphism in enzymes involved in energy production compared to that in nonspecific enzymes in two Drosophila ananassae populations. Proc. Natl. Acad. Sci. U.S.A. 61:582-585.

7. György, P., R. F. Norris, and C. S. Rose. 1954. Bifidus factor. I. A variant of Lactobacillus bifidus requiring a special growth factor. Arch. Biochem. Biophys. 48:193201.

8. Holdeman, L. V., and W. E. C. Moore (ed). 1972. Anaerobe Laboratory manual. Virginia Polytechnic Institute Anaerobe Laboratory, Blacksburg.

9. Johnson, G. B. 1973. Enzyme polymorphism and biosystematics: the hypothesis of selective neutrality. Annu. Rev. Ecol. System. 4:93-116.

10. Johnson, J. L. 1973. Use of nucleic-acid homologies in the taxonomy of anaerobic bacteria. Int. J. Syst. Bacteriol. 23:308-315.

11. Kandler, O., and E. Lauer. 1974. Neuere Vorstellungen zur Taxonomie der Bifidobacterien. Zentralbl. Bakteriol. Parasitenkd. Infektionskr. Hyg. Abt. I Orig. Reihe A 228:29-45.

12. Lowry, O. H., N. J. Rosebrough, A. L. Farr, and R. J. Randall. 1951. Protein measurement with the Folin phenol reagent. J. Biol. Chem 193:265-275.

13. Matteuzzi, D., and F. Crociani. 1973. Urease production and DNA homology in the species Bifidobacterium suis. Arch. Mikrobiol. 94:93-95.

14. Matteuzzi, D., F. Crociani, G. Zani, and L. D. Trovatelli. 1971. Bifidobacterium suis $n$. sp.: a new species of the genus Bifidobacterium isolated from pig feces. $\mathrm{Z}$. Allg. Mikrobiol. 11:387-395.

15. McDonald, J. F., and F. J. Ayala. 1974. Genetic response to environmental heterogeneity. Natura 250: 572-573.

16. Mitsuoka, T. 1969. Vergleichende Untersuchungen über die Bifidobakterien aus dem Verdauungstrakt von Menschen und Tieren. Zentralbl. Bakteriol. Parasitenkd. Infektionskr. Hyg. Abt. I Orig. 210:52-64.

17. Mitsuoka, T., Y. Morishita, and A. Terada. 1974. Actinomyces eriksonii Georg, Roberstadt, Brinkman and Hicklin 1965 identisch mit Bifidobacterium adolescentis Reuter 1963. Zentralbl. Bakteriol. Parasitenkd. Infektionskr. Hyg. Abt. I Orig. Reihe A 226:257-263.

18. Powell, J. R. 1971. Genetic polymorphism in varied environments. Science 174:1035-1036.

19. Powell, J. R. 1975. Isozymes and non-darwinian evolution: a re-evaluation. p. 9-26. In C. Markert (ed.), Isozymes, vol. IV: Genetics and evolution. Academic Press, New York.

20. Reuter, G. 1963-64. Vergleichende Untersuchungen über die Bifidus-Flora im Säuglings- und Erwachsenenstuhl. Zentralbl. Bakteriol. Parasitenkd. Infektionskr. Hyg. Abt. I Orig. 191:486-507.

21. Rogosa, M. 1974. Genus Bifidobacterium, p. 669-676. In R. E. Buchanan and N. E. Gibbons (ed.), Bergey's manual of determinative bacteriology, 8 th ed. The Williams and Wilkins Co., Baltimore.

22. Scardovi, V., and F. Crociani. 1974. Bifidobacterium catenulatum, Bifidobacterium dentium, and Bifidobacterium angulatum: three new species and their deoxyribonucleic acid homology relationships. Int. J. Syst. Bacteriol. 24:6-20.

23. Scardovi, V., and B. Sgorbati. 1974. Electrophoretic types of transaldolase, transketolase, and other enzymes in bifidobacteria. Antonie van Leeuwenhoek J. Microbiol. Serol. 40:427-440.

24. Scardovi, V., B. Sgorbati, and G. Zani. 1971. Starch gel electrophoresis of fructose 6-phosphate phosphoketolase in the genus Bifidobacterium. J. Bacteriol. 106: 1036-1039.

25. Scardovi, V., and L. D. Trovatelli. 1965. The fructose6-phosphate shunt as peculiar pattern of hexose degra- 
dation in the genus Bifidobacterium. Ann. Microbiol. 15:19-29.

26. Scardovi, V., and L. D. Trovatelli. 1969. New species of bifid bacteria from Apis mellifica $\mathrm{L}$. and Apis indica F. A contribution to the taxonomy and biochemistry of the genus Bifidobacterium. Zentralbl. Bakteriol. Parasitenkd. Infektionskr. Hyg. Abt. II 123:64-88.

27. Scardovi, V., and L. D. Trovatelli. 1974. Bifidobacterium animalis (Mitsuoka) comb. nov. and the "minimum" and "subtile" groups of new bifidobacteria found in sewage. Int. J. Syst. Bacteriol. 24:21-28.

28. Scardovi, V., L. D. Trovatelli, B. Biavati, and G. Zani. 1979. Bifidobacterium cuniculi, Bifidobacterium choerinum, Bifidobacterium boum, and Bifidobacterium pseudocatenulatum: four new species and their deoxyribonucleic acid homology relationships. Int. J. Syst. Bacteriol. 29:291-311.

29. Scardovi, V., L. D. Trovatelli, F. Crociani, and B. Sgorbati. 1969. Bifid bacteria in bovine rumen: $B$. globosum n. sp. and B. ruminale n. sp. Arch. Mikrobiol. 68:278-294.

30. Scardovi, V., L. D. Trovatelli, G. Zani, F. Crociani, and D. Matteuzzi. 1971. Deoxyribonucleic acid homology relationships among species of the genus Bifidobacterium. Int. J. Syst. Bacteriol. 21:276-294.

31. Scardovi, V., and G. Zani. 1974. Bifidobacterium magnum sp. nov., a large acidophilic bifidobacterium isolated from rabbit feces. Int. J. Syst. Bacteriol. 24:2934 .
32. Scardovi, V., G. Zani, and L. D. Trovatelli. 1970. Deoxyribonucleic acid homology among the species of the genus Bifidobacterium isolated from animals. Arch. Mikrobiol. 72:318-325.

33. Sgorbati, B., G. Lenaz, and F. Casalicchio. 1976. Purification and properties of two fructose-6-phosphate phosphoketolases in Bifidobacterium. Antonie van Leeuwenhoek J. Microbiol. Serol. 42:49-57.

34. Sgorbati, B., and V. Scardovi. 1979. Immunological relationships among transaldolases in the genus Bifidobacterium. Antonie van Leeuwenhoek J. Microbiol. Serol. 45: 129-140.

35. Sgorbati, B., G. Zani, L. D. Trovatelli, and V. Scardovi. 1970. Gluconate dissimilation by the bifid bacteria of the honey bee. Ann. Microbiol. 20:57-64.

36. Smithies, O. 1955. Zone electrophoresis in starch gels: group variations in the serum proteins of normal human adults. Biochem. J. 61:629-641.

37. Trovatelli, L. D., F. Crociani, M. Pedinotti, and V. scardovi. 1974. Bifidobacterium pullorum sp. nov.: a new species isolated from chicken feces and a related group of bifidobacteria isolated from rabbit feces. Arch. Microbiol. 98:187-198.

38. Trovatelli, L. D., and D. Matteuzzi. 1976. Presence of bifidobacteria in the rumen of calves fed different rations. Appl. Environ. Microbiol. 32:470-473.

39. Zani, G., B. Biavati, F. Crociani, and D. Matteuzzi. 1974. Bifidobacteria from the faeces of piglets. J. Appl. Bacteriol. 37:537-547. 